\title{
Autophagy: Can it become a potential therapeutic target? (Review)
}

\author{
XIAO HONG BAO ${ }^{1}$, YOSHIO NAOMOTO ${ }^{1}$, HUI FANG HAO ${ }^{1}$, NOBUYUKI WATANABE ${ }^{1}$, \\ KAZUFUMI SAKURAMA ${ }^{1}$, KAZUHIRO NOMA ${ }^{1}$, TAKAYUKI MOTOKI ${ }^{1}$, YASUKO TOMONO ${ }^{2}$, \\ TAKUYA FUKAZAWA ${ }^{1}$, YASUHIRO SHIRAKAWA ${ }^{1}$, TOMOKI YAMATSUJI ${ }^{1}$, \\ JUNJI MATSUOKA ${ }^{1}$ and MUNENORI TAKAOKA ${ }^{1}$
}

\begin{abstract}
${ }^{1}$ Department of Gastroenterological Surgery, Transplant, and Surgical Oncology, Graduate School of Medicine, Dentistry, and Pharmaceutical Sciences, Okayama University, Okayama; ${ }^{2}$ Shigei Medical Research Institute, Okayama, Japan
\end{abstract}

Received October 1, 2009; Accepted December 8, 2009

DOI: 10.3892/ijmm_00000369

\begin{abstract}
Autophagy is a cellular lysosomal degradation pathway involved in proteins and organelles recycling for promoting cell survival, development and homeostasis. It is a multistep process and genetic studies have identified many proteins that participate in autophagosome formation and fusion with lysosomes, and various signaling factors that associate with the regulation of autophagy. In general, autophagy acts as a cell protector and its dysfunction is correlated with diverse pathologies, such as neurodegeneration, liver, heart and muscle diseases, cancer, inflammation and ageing. However, its role in cell death increases the complexity of the autophagic degradation system. A broad understanding of autophagy, ranging from detailed processes, including induction, formation and degradation, to function in physiology and pathology, revealed by accumulating studies, may be helpful for formulating therapeutic strategies for autophagyassociated human diseases.
\end{abstract}

\section{Contents}

1. Introduction

2. How autophagy happens

3. What roles does autophagy play in physiology?

4. Autophagy and diseases

5. Conclusion

Correspondence to: Dr Yoshio Naomoto, Department of Gastroenterological Surgery, Transplant, and Surgical Oncology, Graduate School of Medicine, Dentistry, and Pharmaceutical Sciences, Okayama University, 2-5-1 Shikata-cho, Okayama, 700-8558, Japan

E-mail: ynaomoto@md.okayama-u.ac.jp

Key words: autophagy, protein and organelle degradation, homeostasis, protector, physiological and pathological roles, human diseases, therapy

\section{Introduction}

Autophagy is a process of cellular self-digestion that is essential for survival, differentiation, development and homeostasis. Misfolded proteins, superfluous or damaged organelles, and invading microorganisms are digested by lysosomal autophagic-degradation, generating intracellular nutrients and energy for recycling. Several autophagy related genes have been identified in diverse organisms from single cell yeast to multicellular worms, plants, flies and mammals. Most of the genes in yeast are highly homologous with those in higher organisms, suggesting that autophagy is an evolutionary conserved function (1). Based on the physiological function and mechanism, there are at least three types of autophagy, microautophagy, macroautophagy and chaperone-mediated autophagy (CMA). Microautophagy and macroautophagy deliver large structures (e.g. organelles, invasive microbes or bulk cytoplasm) to lysosomes through both selective and non-selective manner, whereas CMA only delivers unfolded substrate protein in a selective manner, chaperone-mediated degradation $(2,3)$. Macroautophagy (hereafter referred to as autophagy) is the most studied form of autophagy, and is the principal basis of this review. Population studies show the complicated roles of autophagy in human health and diseases. A basal level of autophagy is required for cell survival and cellular homeostasis; a disruption of the basal expression level, too little or too much autophagy, may result in certain diseases. Understanding autophagy genesis and its physiological, as well as its pathological functions may provide a rationale for the treatment of autophagy-related diseases in the future and will promote further progress in this field.

\section{How autophagy happens}

The process of autophagosome maturation and related genes. Nutrient deficiency, hormonal factor withdrawal and/or microbial pathogen infection induces autophagy, in which the isolation membranes, autophagosomes, and autolysosomes are formed in order. Atg proteins encoded by AuTophaGyrelated (Atg) genes, which have been thoroughly investigated in yeast, are involved in the process of autophagy (4). 


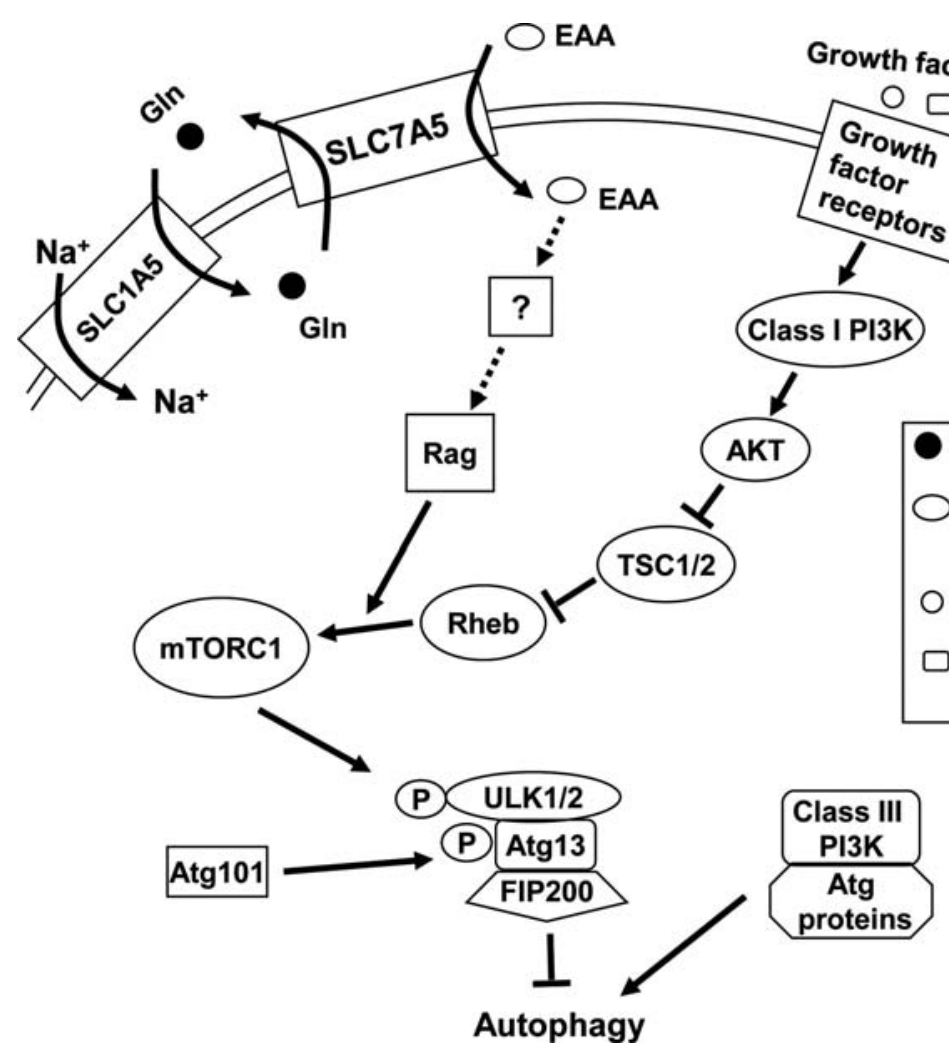

Figure 1. Regulation of autophagy induction. Basal expression of autophagy is enhanced by environmental stimuli including amino acids, growth factors and microbial pathogens. Essential amino acids are transported into the cytoplasm in a glutamine/ $\mathrm{Na}^{+}$-dependent manner. Intracellular amino acids influence unknown factors that activate mTORC1 through the Rag GTPases. Growth factors stimulate the class I PI3K-Rhep pathway and then activate mTORC1. mTORC1 inhibits autophagy via the ULK1/2-Atg13-FIP200 complex, the direct downstream target of mTORC1. Atg 101 is required for the stability and basal phosphorylation of Atg13 and ULK1. Furthermore, class III PI3K is an activator of autophagy. Dashed lines indicate possible regulatory effects.

Autophagy induction. Autophagy is tightly regulated during development (Fig. 1). The presence of extracellular rich nutrients (amino acids) and growth factors (i.e. insulin, insulin-like growth factor) shut off autophagy through the class I phosphatidylinositol 3-kinase (PI3K) (5) and target of rapamycin (TOR) pathways (6). TOR complex 1 (TORC1) performs a key role in mammals during autophagy and it is active in normal growth conditions as a negative regulator of autophagy. Amino acids and especially the essential amino acids are required for its activation. The pathway by which the extracellular amino acids are transported intracellularly, subsequently leading to the activation of mTORC1 has been identified by Nicklin et al (7). Extracellular L-glutamine, non-essential amino acid, is transported into cells in a sodium $\left(\mathrm{Na}^{+}\right)$-dependent manner via Solute carrier family 1 member 5 (SLC1A5). Intracellular L-glutamine acting as an efflux substrate is used for regulating the import of extracellular L-leucine through the heterodimeric Solute carrier family 7 member 5/Solute carrier family 3 member 2 (SLC7A5/ SLC3A2) bidirectional transporter. In cytoplasm, transported amino acids activate mTORC1 via the Rag GTPases (a family of four related small guanosine triphosphatases) to re-localize mTORC1 in the same intracellular compartment that contains its activator Ras homolog enriched in the brain (Rheb) (8). Conversely, when cells are deprived of glutamine, leucine cannot be imported, resulting in the inactivation of mTORC1 and the induction of autophagy (7). For the response of cells to growth factors, the extracellular fragments of growth factor receptors bind to growth factors, leading to the activation of the intracellular tyrosine kinase of the receptor. The activation form of growth factor receptors induce the class I PI3K/AKT pathway following mTOR activation that inhibits autophagy (9). Compared with the inhibitory functions of class $1 \mathrm{PI} 3 \mathrm{~K}$ to autophagy, class III pI3K activates autophagy (5).

Although it is well known that mTOR is a key negative regulator of autophagy, the exact molecular mechanism by which mTOR regulates autophagy is still unclear. Several studies have reported the transduction of mTOR signaling associated with the ULK-Atg13-FIP200 complex ( 3-MDa protein complex) (ULK, the mammalian Atg1 homologue; Atg13, the mammalian Atg13 homologue; FIP200, focal adhesion kinase family interacting protein of $200 \mathrm{kDa}$ ), which is an essential part of the regulatory protein complex of autophagy (10-12). In nutrient rich conditions, mTOR phosphorylates $\operatorname{Atg} 13$ and ULK (including ULK1 and ULK2) inhibits the kinase activity of ULK to FIP200 mediated by Atg13, resulting in the inhibition of the formation of autophagosome. In starvation conditions, however, the phosphorylation of Atg13 and ULK by mTOR is inhibited, Atg13, ULK, and FIP200 are phosphorylated by the activated kinase activity of ULK to promote autophagosome formation (11). These studies show that mTOR regulates autophagy mediated by the ULKAtg13-FIP200 complexes in a nutrient-dependent manner. ULK-Atg13-FIP200 is a direct target of TOR in mammals. Furthermore, a novel mammalian autophagy protein Atg101 has been identified and it causes the induction of autophagy by interacting with Atg13, ULK and FIP200 (13) (Fig. 1). 


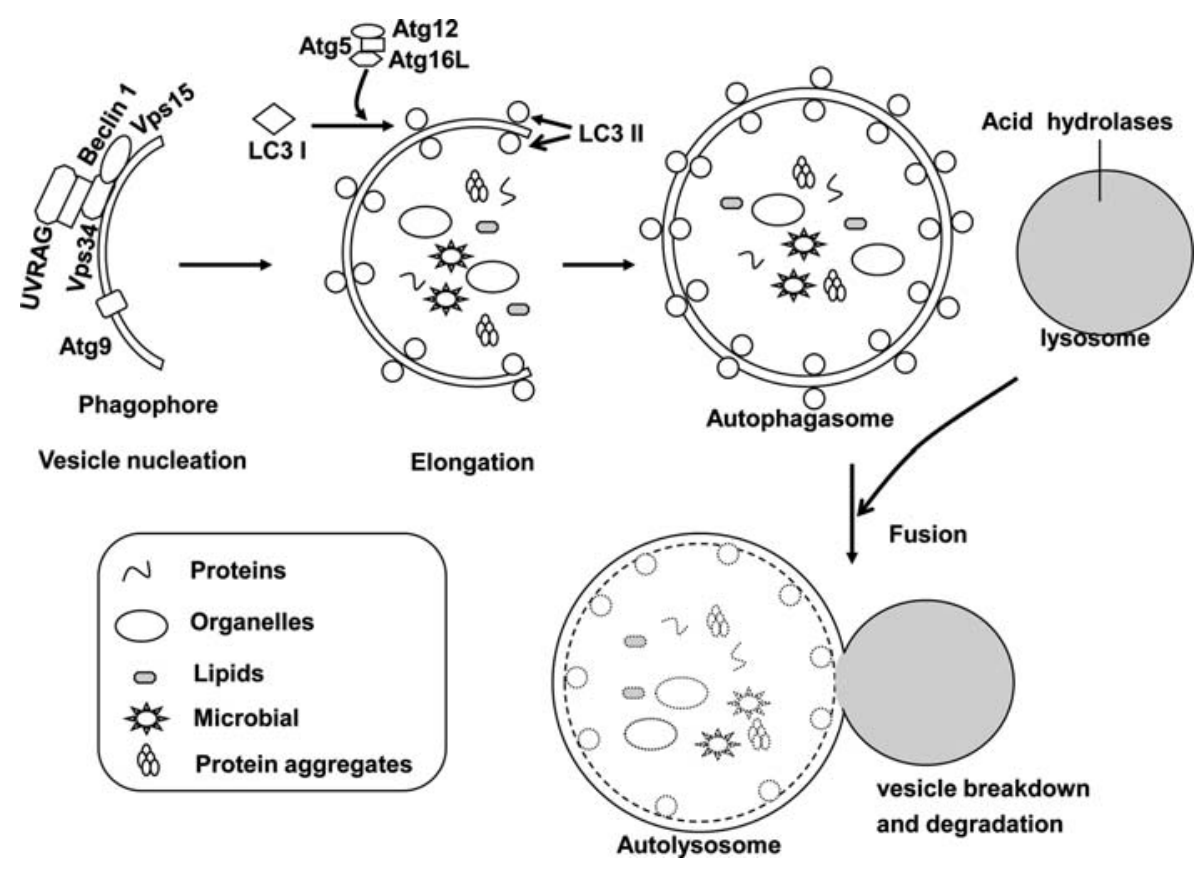

Figure 2. The process of autophagosome formation and degradation. Non-required proteins and their aggregates, organelles, lipids and microbial pathogens are the substrates of the autophagic-lysosomal digestion system. The process of autophagy consists of four stages: vesicle nucleation (initiation of the formation of the phagophore), vesicle elongation, docking and fusion (autophagosome maturation and fusion with lysosome), and final degradation. The main complex systems during autophagy are the LC3 conjugation system and the Atg12-Atg5-Atg16L complex. Also, many other proteins are involved in each step of the process of autophagy.

Autophagosome formation and degradation. After induction, the autophagy machinery begins to assemble. The cellular event during autophagy can be dissected into four stages: vesicle nucleation, vesicle elongation, docking and fusion, and vesicle breakdown and degradation (Fig. 2) (14). This is a comparatively perplexing process and involves many Atg and other proteins.

Vesicle nucleation refers to the formation of phagophore or isolation membrane (a lipid bilayer structure) from a phagophore assembly site or the pre-autophagosomal structure (PAS), localized in the peri-vacuolar region, in response to starvation conditions (15). Most Atg proteins recruit to the PAS, as analyzed by fluorescence microscopy, and facilitate the formation of phagophore (16). Beclin 1 (yeast Atg6), UVRAG (yeast Vps38), Vps34 and Vps15 are assembled to form a lipid kinase signaling complex to mediate vesicle nucleation (14). Recently, mammalian Atg9, a multi-transmembrane protein, has been reported to be required for the formation of phagophore and plays a crucial role in lipid membrane transport to PAS by self-interaction $(17,18)$. However, the origin of PAS is not well understood in mammals, or even in yeast.

The molecules to be digested are sequestered by the elongated membrane after the formation of the isolation membrane (Fig. 2). This process is called vesicle elongation. It involves two ubiquitin-like conjugation systems, mammalian LC3 (yeast Atg8) and Atg12, among which the major one is LC3 conjugated with phosphatidylethanolamine (PE) (14). The C-terminal proteolysis of LC3 is processed by Atg4, based on the residues Phe 80 and Leu82 of LC 3 that may be recognized by Atg4 (19), and immediately follows synthesis to yield a soluble form, LC3-I. LC3-I is converted to a membrane bound form, LC3 II, through a ubiquitin-like reaction involving Atg7, a ubiquitin-activating enzyme (E1)-like enzyme, and Atg3, a ubiquitin-conjugating enzyme (E2)-like enzyme (20).
LC3 is conjugated to Atg3 via Atg7 and then transferred from LC3-Atg3 (I form) to LC3-PE (II form) through the interaction between Atg7 and Atg12 that forms an Atg16L complex, Atg12-Atg5-Atg16L, which allows the LC3 recruitments to the membrane proximal with PE (21).

The isolation membranes elongate and curve until their leading edges merge to envelope portions of the cytoplasm and form the double membrane autophagosome. LC 3s are located in both the inner and outer membrane of the forming autophagosome and may play an important role in the closure of the autophagosome (22). One possible mechanism by which LC3s are involved in closure is that lipidated LC3s on the isolation membrane may have the function of lateral diffusion, leading LC3-PE to traverse the narrowing leading edge and tether other LC3-PE to close the autophagosome (22). However, there is no direct evidence to demonstrate this hypothesis. Based on the fact that LC3-PE binds to the autophagosomal membrane during autophagy, approaches for detecting LC3 (LC3 II) (generation or localization) have been developed to detect and manipulate the autophagy pathway. Notably, when observing the localization of LC3, the punctate dots do not always reflect the autophagosome due to LC3s aggregate in an autophagy-independent manner. Therefore, the interpretation of LC3 localization should be made carefully (23). After their formation, the outer membranes of autophagosomes fuse with multivesicular lysosomes (called autolysosome), while the inner membranes and sequestrated contents are subsequently digested into amino acids by lysosomal hydrolases within the lysosome lumen. Lysosomal transmembrane proteins such as lysosomalassociated membrane protein 2 (LAMP-2) (24), CLN3 (25) and Rab7 (26) (a small GTP binding protein), rather than Atg proteins, are required for this fusion. At the vesicle degradation and breakdown step, lysosomal cysteine proteases, cathepsins B, 
$\mathrm{D}$, and $\mathrm{L}$ are required for the degradation of the sequestrated contents (14). LC3 and other autophagosome components are also degraded and diminished.

Taken together, the maturation and degradation of autophagosome and autolysosome undergo the complicated process (Fig. 2), regulated by many Atg and other autophagy associated proteins. Physiologically, autophagy occurs at a low basal level, which is enhanced during starvation as well as the accumulation of non-required cellular components, to execute its homeostatic mission. Pathologically, however, dysfunctions of autophagy are the cause of several diseases.

\section{What roles does autophagy play in physiology?}

Maintenance of cellular homeostasis. Autophagy rids the cell of misfolded protein and organelles (eg. mitochondria, peroxisomes, ER and intact intracellular microorganisms) over time, prevents the accumulation of unwanted components and, at least in part, removes intracellular pathogens (Fig. 2). Such functions maintain cellular homeostasis and probably underlie the roles of autophagy in cell survival and in protection against cancer, neurodegeneration, ageing and infection (14). Studies of the tissue-specific Atg gene deletion demonstrate the essential function of basal level autophagy on intracellular protein quality. In Atg5 deleted mice, diffused and abnormal proteins accumulate and aggregate to form microscopically visible structures known as inclusions, which can disrupt the neural system (27). Some protein inclusions may protect cells by sequestering toxic protein and delaying protein degradation or alternatively may inhibit the proteosome and induce cytotoxity under which autophagy as a compensatory mechanism degrades ubiquitin-positive abnormal protein accumulation $(28,29)$. Autophagy may selectively turn over ubiquitin-positive inclusions. In this process, LC3 on the autophagosome may play a vital role in the selective degradation of p62/A170/SQSTM1, which is a ubiquitin-binding protein and involved in the formation of inclusions, due to its direct interaction with p62 (30).

The quality control of highly complex cytosolic cargo, including mitochondria, ribosomes and peroxisomes, is important to maintain proper cellular homeostasis. Because of their ability to sequester structures with varying sizes and geometries, autophagosomes can efficiently clear superfluous and damaged mitochondria, peroxisomes and ribosomes to control the proper number and quality of this cargo. Increasing evidence suggests that the degradation of organelles by autophagy may not be in a random, nonselective fashion, instead, in a selectively targeted pathway. For instance, time-lapse confocal imaging directly visualizes the progression of mitophagy (autophagy degradation of mitochondria) in hepatocytes derived from transgenic GFP-LC3 mice in response to nutrient deprivation; green-fluorescing GFP-LC3s envelope and sequester red-fluorescing mitochondrion (31). The molecular mechanism of this process is unclear. In mammals, however, a BH3-only member of the Bcl-2 family, BNIP3L/NIX, was reported to be required for selective autophagic elimination targeting mitochondria in erythroid maturation (32). Similar to mitophagy, the autophagic degradation of peroxisomes, a normally long-lived cytoplasmic organelle, is commonly termed pexophagy. When the cyto- plasmically exposed $\mathrm{C}$ terminus tail of PMP34, which is a multi-transmembrane integral membrane protein of peroxisome, was linked to mono-ubiquitin moiety, the peroxisome became a selective target of autophagic degradation in mammalian cells. Further experiments showed that pexophagy requires the ubiquitin-binding protein $\mathrm{p} 62$, similar to ubiquitinated protein aggregates, suggesting that this process involves ubiquitin and p62 in the selective autophagy of peroxisomes (33). One recent study showed that GFP labeled ribosome proteins are targeted to the vacuole by ubiquitination during starvation, proposing an autophagy selective elimination of ribosomes (referred to as ribophagy) in yeast (34). Currently, there is no report involving the process of autophagic degradation of ribosomes in mammalian cells. Furthermore, it is reported that autophagy affects the lipid metabolism and regulates intracellular lipid stores (macrolipophagy) by an unknown mechanism (35). More work therefore is needed to explore the process and molecular mechanism in detail.

Autophagy acts as a cyto-protector or a cyto-killer. It is well known that autophagy is essential for survival upon starvation in all eukaryotic organisms from yeast to mammal. In response to different types of stress, including nutrient and growth factor depletion, endoplasmic reticulum (ER) stress, hypoxia, microbial infection, and diseases characterized by the accumulation of protein aggregates, autophagy controls a stress adaption pathway that promotes cell survival (36-39). Autophagy degradation supplies amino and fatty acids that are required for recycling and cellular bioenergetics (ATP). In growth factor-deprived autophagy-inhibited cells, the addition of methylpyruvate, a tricarboxylic acid cycle (TCA) substrate, maintains ATP and cell survival, otherwise the cell viability declines and dies (40). Therefore, ATP production may be through TCA catabolism in which the raw materials are mainly supplemented by autophagy in growth factor withdrawn cells. In addition to the essential role of autophagydependent ATP production in cell survival, a novel biological role in the clearance of apoptotic corpses has been reported during embryonic development (41). Restoration of normal ATP levels by methylpyruvate in autophagy gene deficient (Atg $5^{-/-}$and beclin $1^{-/-}$) embryos is involved in the reversal of the defects in apoptotic cell engulfment, LPC secretion and PS exposure, strongly suggesting that autophagy promotes apoptotic cell corpse clearance that associates with the generation of energy-dependent engulfment signals (41).

Consistent with experiments in vitro, increasing studies in vivo also demonstrate the indispensible cytoprotective role of autophagy. For example, autophagy is transiently increased in normal newborn mice after birth under physiological conditions. A possible explanation is that during neonatal starvation, neonates underwent selfnourishment by inducing autophagy to generate adequate metabolic substrates. Deletion of the autophagy gene (Atg5) results in early neonatal lethality due to amino acid insufficiency (42). A similar study was done by Komatsu et al (43). They established an Atg7 conditional knockout mice model, in which the Atg conjugation system and autophagosome formation were impaired, also showed evidence of autophagy function in cell survival and development. Atg $7^{-/-}$ 
neonates died within 1 day after birth and under starvation conditions died earlier than wild-types after caesarean delivery, suggesting that the inability of these mice to is due to the critical autophagy related protein Atg7 deficiency. Based on these studies, in physiology, autophagy can promptly meet the cellular demands for metabolic substrates in response to metabolic stress by the transformation of intracellular nutrients and energy resources. Impairment of this protein recycling system might disrupt cell survival and development. This may explain the reduced protein synthesis and developmental defects in autophagy null mouse embryos during preimplantation development, in which fertilization of autophagydeficient (Atg $5^{-/-}$) oocytes with autophagy-deficient (Atg $5^{-/}$) sperm did not develop and remained at the four- or eight-cell stage, but those with wild-type sperm developed (44).

Autophagy also regulates innate and adaptive immune systems. This functions through the killing of intracellular pathogens, antigen presentation, tolerance and lymphocyte development, type I interferon production, as well as the negative regulation of cytokine signaling and inflammation (45). Autophagy can selectively target microorganisms, including viruses, bacteria and parasites (free microbes in the cytosol or pathogen-containing phagosomes), and deliver them to lysosomes for hydrolysis. With respect to the innate defense system, a representative example of autophagy targeting intracellular microbes is the elimination of group A Streptococci (GAS), which escapes from endosomes into the cytoplasm and then are killed by autolysosomes in wild-type cells, but are released from autophagy-deficient Atg5 $5^{-/-}$cells (46). Endogenous antigen presentation to the adaptive immune system is also performed by autophagy. For example, endogenous Epstein-Barr virus nuclear antigen 1 (EBNA1) (47) and Mycobacterium tuberculosis (Mtb) 85B antigen (48) are processed for MHC (major histocompatibility complex) class II presentation to $\mathrm{CD}^{+} \mathrm{T}$ lymphocytes. BCG vaccine efficacy is enhanced by autophagy through increasing mycobacterial antigen Ag85B presentation in a mouse model further demonstrates autophagic enhancing immunity (49).

However, an apparent paradox of autophagy is that it acts upon both cytoprotection and cytotoxicity. The death induced by autophagy is defined as non-apoptotic or type II programmed cell death and possesses the characteristic of the increased autophagosomes without any signature of apoptosis or necrosis (50). However, when a dying cell shows features of several types of death (e.g. autophagy and apoptosis), it is difficult to identify if the cell is dying with autophagy or through autophagy. If cell death accompanies autophagy, it means autophagy may be induced by some death events rather than being an executor of cell death. If the cell is dying through autophagy, autophagy may be a part of the apoptotic program or be in parallel with apoptosis for triggering cell death (50). The first evidence that autophagy contributes to cell death (independent of apoptosis) was that the embryonic fibroblasts (MEF) from Bax/Bak double knockout mice, which were resistant to apoptosis, were treated with various apoptotic stimuli, leading to non-apoptotic cell death that associated with the generation of autophagosomes/autolysosomes, the accumulation of Beclin 1 and APG5-APG12 complex, and the overexpression of Bcl-2/Bcl-xL (51). In that case, the antiapototic Bcl-2/Bcl-xL may promote autophagosome formation by the regulation of beclin 1 . Nevertheless, the opposite role of the Bcl-2 family in autophagy was reported. The interaction between Bcl-2 and beclin 1 inhibits autophagy to sustain the physiological functions of autophagy, whereas if Bcl-2 cannot bind to beclin 1, an overphysiological range of autophagy may promote cell death. This antiautophagic function of Bcl-2 possibly maintains autophagy at the basal level being more compatible with cell survival than cell death (52). Different stress conditions, at which the former is apoptosis-induced autophagy but the latter is starvationinduced autophagy, might be the reason for the converse roles of the Bcl-2 family. Bcl-2 might function more generally under starvation conditions but this still remains to be further identified.

Although autophagy plays a role in cell death in certain settings, there is no report that autophagy related genes promote programmed cell death in physiological in vivo or that the down-regulation of autophagy genes avoid cell death. On the contrary, Atg5 or beclin 1 deficiency in embryonic cells increase the apoptotic cells and the cells undergo apoptotic death during development (41). The removal of apoptotic cells (apoptotic bodies), the final step of apoptosis, may require autophagy, based on the fact that autophagy null embryoid bodies fail to generate an 'eat me' signal (phosphatidylserine exposure) and secrete low levels of 'come and get me' signal (lysophosphatidylcholine). Interestingly, the 'come and get me' signal in apoptotic cells is also expressed by sphingosine 1phosphate (S1P) (53), which was reported to be associated with autophagy formation (54).

Many studies have shown autophagy, which primarily acts as a cell guardian, and apoptosis, which unchangeably leads to cell death, share common regulatory factors and one can be regulated by the other (39). Both apoptosis and autophagy lead to cell death via death-associated protein kinase (DAPK), BNIP3 and ceramide. Viewed from several signaling pathways that associate with autophagy and/or apoptosis: Beclin 1, BH3only protein BAD, the lysosomal protein DRAM, the nuclear form of p53 (p53n), and Sestrin2 are the positive effectors of autophagy, whereas mTORC1, mTORC2, PKB, Bcl-2, Bax/Bak, the cytoplasmic form of p53 (p53c), the transcription factor $\mathrm{NF}-\kappa \mathrm{B}$, inositol 1,4,5-trisphosphate receptor [IP(3)R] are the negative ones (see details ref $50,55,56$ ). The factors that inhibit apoptosis also inhibit autophagy. For example, antiapoptotic Bcl-2 inhibits beclin 1 (52). Notably, the factors that stimulate apoptosis can also induce autophagy. For instance, pro-apoptotic $\mathrm{BH} 3$-only protein BAD activates autophagy by removing Bcl-2 inhibitory interaction with beclin 1 (57). Also, more apoptosis-sensitized cells are likely to die via autophagy if autophagic protection of these cells cannot withstand unanticipated, unfavourable stress circumstances. This intricate molecular interplay between autophagy and apoptosis must be fully elucidated by further studies and might be a mirror of the complex relationship of autophagy with human disease pathogenesis.

\section{Autophagy and diseases}

As mentioned above, a basal and constitutive level of autophagy is indispensible for intracellular homeostasis and quality control for healthy individuals. Mounting evidence has demonstrated that disruption of its physiological function is 
strongly related to human diseases, including neurodegeneration, cancer, liver and heart diseases, ageing, myopathies, and infections (58). This section will discuss the current knowledge of autophagy-related diseases and their potential therapeutics.

Autophagy-related neurodegenerative diseases. Neurodegenerative diseases such as Alzheimer's disease, amyotrophic lateral sclerosis, Parkinson's disease, prion diseases, Huntington's disease and spinocerebellar ataxias, have the common characteristic of protein aggregation (59). For example, $\beta$-amyloid and tau aggregates accumulate in Alzheimer's disease. Expanded polyglutamine (PolyQ) stretch caused by the expansion of CAG trinucleotide repeat within several gene exons seen in Huntington's disease and several types of spinocerebellar ataxias. $\alpha$-synuclein fibrils linked to familial Parkinson's disease self-aggregate and deposit in the brain $(60,61)$. These protein aggregates, the neurotoxic components, are substrates of the autophagy degradation system. Boland et al suggested that the basal expression of autophagy is crucial in primary cortical neurons and there is no large amount of autophagosome accumulation in healthy brains because of efficient lysosomal hydrolysis of newly formed autolysosomes (62). Therefore, it is true that autophagy plays a role in the protection against neurodegeneration. However, in patients with diverse neurodegenerative diseases, the autophagic clearance system may reach saturation and thereby become unable to eliminate excess aggregate-prone mutant proteins, or dysregulation or defection may occur in the autophagy pathway. Impaired autophagic vacuoles (AVs) clearance during autophagosome transport to lysosomes or during substrate degradation within the lysosome may lead to AVs accumulation with morphologies that are observed in Alzheimer's disease (AD) (62). Contrary to the beneficial effect of basal autophagy, an excess accumulation of autophagosomes promotes neuronal cell loss under certain circumstances, such as dysfunctional endosomal sorting complex required for transport III (ESCRT-III), which is associated with frontotemporal dementia (63). This implies that either excessive autophagosome synthesis or reduced autophagosomelysosome fusion may contribute to the pathogenesis of neurodegeneration by the dysfunctional autophagic process.

Owing to the protective role of autophagy in neuron systems, it may be a therapeutic target against neurodegenerative diseases. Upregulation of autophagy may improve the clearance of neurotoxic aggregate-prone proteins. The inhibition of TOR, a negative regulator of autophagy, by rapamycin or its analogue CCI-779 is protective in Drosophila and mouse models of Huntington disease (64). mTOR is required for various cellular events, including protein synthesis (e.g. the initiation of mRNA translation, ribosome biogenesis, transcription), cell growth and proliferation, and cytoskeletal reorganization (65), but its inhibition by rapamycin causes some adverse effects such as cell cycle arrest, poor wound healing, and mouth ulcers (66). Small molecule enhancers of rapamycin (SMERs), however, induce autophagy in an mTOR independent mechanism. They enhance the elimination of mutant huntingtin and $\alpha$-synuclein, and protect against neurodegeneration in the Huntington's disease model of Drosophila (67). Recently, autophagy induction by a group of new drugs, L-type $\mathrm{Ca}^{2+}$ channel antagonists, $\mathrm{K}^{+}$ATP channel opener minoxidil, and Gi signaling activator clonidine in an mTOR independent pathway were reported in Huntington's disease mammalian cell, fly and zebrafish models (68).

Nevertheless, it is important to understand the pathology underlined by autophagy in different neurodegenerative diseases. Pharmacologically enhancing autophagosome formation in $\mathrm{AD}$ where the autophagosome clearance system is defective may exacerbate the accumulation of the unmatured autophagosomes, some of which may generate toxic proteolytic product $A B$ and/or others $(62,69)$. Therefore, improvement in the late stages of the autophagy process may be the best therapeutic modulation of autophagy in AD. In addition, the inhibition of autophagy by small molecules, such as 3-MA, might be promising therapeutic strategies for the early stages of some forms of FTD or other neurodegenerative diseases where excess produces autophagosomes (63).

Autophagy in liver, heart and muscle diseases. The basal level of autophagy plays an important role in protein and organelle quality control in several tissues such as the liver, heart and muscles. Once absent of autophagy activation, the tissues induced are to form certain protein aggregates that are physiologically cytotoxic and thereby lead to occurence of diseases. In $\alpha 1$-antitrypsin deficiency, which causes severe chronic liver injury and hepatocellular carcinoma, mutant $\alpha 1$-antitrypsin Z ( $\alpha 1-\mathrm{ATZ})$ proteins accumulate to form aggregates within the endoplasmic reticulum (ER) of the liver cells (70). Deletion of Atg5 in vitro hampered the degradation of $\alpha 1$-ATZ, especially inclusions of $\alpha 1-\mathrm{ATZ}$ accumulated; transgenic liver-specific expression of $\alpha 1-\mathrm{ATZ}$ in vivo is sufficient to induce mouse hepatocyte autophagy, suggesting that the protein quality control function of autophagy in important in $\alpha 1-\mathrm{ATZ}$ and its degradation function which protects liver cells from the cytotoxicity of $\alpha 1-\mathrm{ATZ}$ accumulation. Based on this, autophagy augmentation by pharmacological agents would likely be beneficial in this setting.

Similar to the neuron and liver, basal autophagy is also required for protein and organelle control in normal cardiac function and invalidity in this process results in cardiac dysfunction and heart failure. Abnormal protein aggregation and ubiquitinated protein accumulation can be detected in human hearts with idiopathic or ischemic cardiomyopathies (71-73). In a mouse model of load-induced heart failure, autophagy, probably triggered by protein aggregation, sequestered a high density of ubiquitinated protein aggregates (74). Also, knockout of cardiac-specific Atg5 in adulthood results in heart hypertrophy and contractile dysfunction that is accompanied by elevated ubiquitinated proteins, sarcomere and abnormal mitochondria accumulation. Thus, stress-induced upregulation of autophagy provides sufficient energy substrates and enhances ventricular remodeling, playing an adaptive role (75). However, opposite to this adaptive role, cardio-myocyte autophagy is maladaptive in certain settings based on the experimental outcome where contractile function was maintained and not handicapped in a beclin $1^{+/-}$ mice model in a pressure overload setting (76). Perhaps, it is necessary to clarify whether the contradictory effects of cardiomyocyte autophagy are induced by different types of protein aggregates that form and/or the associated autophagic response (77). 
The pathogenesis of several muscle diseases like Danon disease and Pompe disease may be associated with an impairment in autophagosome-lysosome fusion. Danon disease, an extremely rare X-linked dominant disorder characterized by cardiomyopathy, myopathy, and variable mental retardation, is caused by the mutation of LAMP-2 (78). The failure of autophagosome to fuse with lysosome leads to large amount of autophagosomes accumulating in the muscles of LAMP-2deficient mice and patients. The impairment of autophagosomallysosomal fusion also contributes to the accumulation of autophagic substrates, including potentially toxic aggregateprone ubiquitinated proteins (e.g. glycogen), in the core of the Pompe myofibers that occur in Pompe disease, and the deficiency of glycogen-degrading lysosomal enzyme acid $\alpha$-glucosidase (GAA) (79). Ramachandran et al recently uncovered the molecular mechanism of X-linked myopathy with excessive autophagy (XMEA) that is characterized by progressive vacuolation and atrophy of skeletal muscle (80). $V M A 21$ gene deficiency is the associated with autophagic myophathy and it reduces lysosome's ability of degradation and inhibits autophagy by increasing lysosomal $\mathrm{pH}$. This leads to an accumulation of large and ineffective autolysosomes and finally vacuolates the cell and atrophies the tissue. These data suggest that regardless of the diseases from the liver, heart, or muscle, aggregate-prone proteins that are mainly cleared by autophagy are involved in the pathology of these diseases, and thereby autophagy upregulation is helpful for toxic protein clearance.

Autophagy in cancer. Cancer is genetically linked to autophagy defection and several Atg genes or genes that regulate/execute the autophagy pathway play a role in tumor suppression or oncogenesis (81). The monoallelic deleted autophagic gene beclin 1 was identified in human breast, ovarian, prostate cancers and brain tumors, suggesting the importance of this event in tumorigenesis $(82,83)$. Mice with heterozygous disruption beclin 1 had a reduction of autophagy and the development of spontaneous tumors including lymphomas, lung carcinomas, hepatocellular carcinomas, and mammary precancerous lesions $(84,85)$. Transplantation of immortalized kidney and mammary epithelial cells derived from beclin 1 heterozygous-deficient mice were more tumorigenic compared with that of those derived from wild-type mice (82). These studies provide compelling evidence that beclin 1 is a haploin sufficient tumor suppressor. Other essential autophagy mediators such as ultraviolet irradiation resistance-associated gene (UVRAG), Bif-1 and Ambra1, which interact with Bcl-2 to activate Vps34 (the class III phosphatidylinositol-3-kinase), may also inhibit cell proliferation and/or tumorigenesis (81). More recently, the frameshift mutation in which Atg genes with mononucleotide repeat including Atg2B, Atg5, Atg9B and Atg12 were at a high frequency in gastric and colorectal cancers with microsatellite instability, revealing that these mutations possibly contribute to cancer development. This means that these genes have an effect on tumor suppression (86). To further confirm this function of Atg genes, it might be better to establish an animal model with Atg knockdown or knockout. Atg $4 c$ encoding a cysteine proteinase involved in the processing and delipidation of LC3 has been proposed to contribute to tumor suppression according to the experimental results that fibrosarcomas increased by chemical agent in $\operatorname{Atg} 4 \mathrm{C}^{-/-}$mice (87). Therefore, it is rational that genes at different steps of autophagy also function in tumor suppression. However, some autophagy regulator genes are known as oncogenes. Oncogenes encoding class I PI3K, AKT, mTOR, and Bcl-2 are the negative regulator of autophagy, whereas tumor suppressor genes encoding p53, PTEN, DAPk, TSC1/ TSC2, BH3 - only proteins and drs are the positive regulators of it $(88,89)$.

The molecular mechanism by which autophagy acts as a tumor suppressor is not so clear. However, more recently, Mathew et al have reported that p62 may be the key mediator in autophagic suppression of tumors (90). Sustaining the expression of p62 is a common feature of tumor cells in which autophagy is deficient and it is associated with oxidative stress activation through $\mathrm{NF}-\kappa \mathrm{B}$ and tumorigenesis. The failure to eliminate P62 in autophagy-defective beclin $1^{+/-}$and Atg $5^{-1-}$ iBMK cells also leads to increased DNA damage and genomic instability. In other words, under metabolic stress, autophagy may promptly recycle p62 preventing p62-induced genomic instability and tumorigenesis. This may strongly validate one of the hypotheses, proposed by White and colleagues, that explained that the loss of autophagy, a cell survival pathway, promotes oncogenesis possibly due to genomic instability in metabolically stressed cells (91). Therefore, autophagy promotion for the efficient clearance of p62 might be a strategy for cancer chemoprevention in patients with hepatocellular carcinomas where p62 is commonly upregulated.

One complicated aspect of autophagy targeting cancer therapy is that autophagy inhibition, rather than autophagy activation, may enhance the tumor cell sensitization to cytotoxic agents (88). In this situation, the pro-survival effect of autophagy, rather than the tumor suppressor effect, contributes to tumor cells preventing death induced by chemotherapy or radiotherapy. For example, autophagy induction via ATF4 may be an important resistance mechanism in Bortezomibtreated breast cancer cells (92). Autophagy activation may protect HER2-overexpressing breast cancer cells from trastuzumab toxicity (93). Pharmacological and genomic inhibition of autophagy have been utilized in several experimental cancers, such as colon cancer sensitized to chloroquine (94), breast cancer killed by the estrogen receptor antagonist tamoxifen (95), pharyngeal, breast, and lung cancers, cervical squamous cell, and rectum carcinomas sensitized to $\gamma$-irradiation (96). Taken together, at the early stages of the tumor, autophagy functions as a tumor suppressor, under which autophagy induction may be effective in preventing tumor formation and progression. During tumor progression, however, autophagy acts as a tumor growth promoter, under which autophagy inhibition may enhance the cytotoxic agents efficacy and tumor regression.

Autophagy and diseases in the immune system. Autophagy can directly eliminate pathogens, protect infected cells, and also mediate trafficking events required for innate and adaptive immunity (45). Although many pathogens such as bacteria and viruses are removed by autophagy, it is unavoidable that some pathogens have developed strategies to outsmart autophagy. There are three possible mechanisms by which bacteria escape: inhibiting autophagosome fusion with lysosomes, blocking compartments degradation after fusion with lyso- 
somes, or subverting autophagosomes to their own replicative advantage. The bacterium Shigella flexneri escapes from autophagosome engulfment through secreting IcsB protein which blocks $S$. flexneri recognition by the autophagic machinery (97). Listeria monocytogenes can replicate in spacious Listeria-containing phagosomes (SLAPs), which are non-acidic and nondegradative compartments and formed in an autophagy-dependent manner. Bacterial Listeriolysin O, a pore-forming toxin essential for L. monocytogenes virulence, is required for SLAP formation. These suggest that listeriolysin $\mathrm{O}$ may inhibit autophagosome acidification by creating pores and therefore prevent lysosomal degradation in SLAPs (98). In some settings, microviral invasion may be important for microbial pathogenesis. Studies on herpesviruses provide the most compelling evidence for this presumption. The $\alpha$ herpes simplex virus type 1 (HSV-1) encodes the neurovirulence protein ICP34.5 that binds to Beclin 1 and inhibits autophagosome initiation through this interaction (99). The $\gamma$ herpes viruses, Kaposi sarcoma-associated herpesvirus and murine $\gamma$-herpesvirus 68, however, inhibit autophagy at the initiation step by expressing the viral $\mathrm{Bcl}-2(\mathrm{v}-\mathrm{Bcl}-2)$ that also blocks the function of beclin 1 via binding (100). In these settings, it may be feasible in antimicrobial therapy to selectively disrupt the interactions between microbial virulence factors and their target in host autophagy proteins.

Several autophagic genes have been reported to associate with inflammatory diseases. Functional knockdown of Atg16L1 that is expressed in intestinal epithelial cell lines abolished autophagy of Salmonella typhimurium (101). The distinct expression patterns of IRGM gene encoding autophagystimulatory immunity-related GTPase were identified in Crohn's disease, an inflammatory bowel disease (102). Reduced expression of IRGM significantly compromised the efficiency of anti-bacterial autophagy, but a modest overexpression of IRGM protein enhanced the autophagy of Salmonella. These studies support a role of Atg16L1 and IRGM in anti-bacteria autophagy. The genome-wide association scan studies in Crohn's disease demonstrated single-nucleotide polymorphisms in Atg16L1 and IRGM and were strongly associated with the susceptibility to this disease $(101,103,104)$. This suggests that the dyregulation of autophagy may contribute to the pathogenesis of Crohn's disease. However, there is no evidence that these mutations result in autophagy impairment. At least the T300A Atg16L1 variant is not a loss-of-function mutant. Therefore, it might be possible that these polymorphisms disrupt specific autophagic innate defensive system in gut commensals, causing inflammation after microbion invasion, or compromise autophagic tolerance through selfantigen presentation on MHC class II molecules on the mucosal surface, which is then easily broken by proinflammatory microbial stimuli at this site (45).

Autophagy in ageing. A common feature of all aged cells is the intracellular accumulation of damaged proteins and organelles that leads to the decreased ability of the organism to survive (105). The decreased protein degradation plays an important role in ageing by the reduced breakdown of altered proteins and life-span prolongation of proteins in cells that consequently is post-translationally altered. During this process, the defection of the autophagic/lysosomal proteolytic system may be the main cause of reduced protein degradation, since the proteolytic system - proteasome can not digest large molecules such as impaired organelles. Indeed, there is evidence of a gradual reduction in autophagy with age $(105,106)$. Autophagy deficiency can lead to neurodegenerative diseases (27) and heart dysfunction (75) in experimental models, and hence it is supposed that decreased autophagic activity with age contributes to the functional deterioration of ageing organisms. Therefore, autophagy activation may protect organisms from ageing due to the increased ability to get rid of damaged proteins and organelles. Caloric restriction, the only intervention for delaying ageing and expanding life span, reverses autophagy decline with ageing possibly because of reduced insulin/IGF-1 signaling that inhibits autophagy (107). Fasting can promote longevity, but it may cause potentially adverse effects of caloric restriction on human health and hence currently, alternative approaches are being studied that mimic the benefit effects of caloric restriction. The application of antilipolytic drugs that increase autophagy and extend longevity is a good example $(108,109)$.

The molecular basis for age-related decline in the autophagic proteolytic system remains to be clarified. However, the factors that regulate the ageing process are notably interrelated with the autophagy pathway (reviewed in ref. 110). For instance, insulin/IGF-1 signaling, in which the IGF-1 receptor may be a central regulator of longevity in mammals (111), inhibits autophagy by PI3K and mTOR signaling. The forkhead family transcription factor FoxO, a key downstream target of insulin, growth factor, nutrient, and oxidative stress stimuli, plays a role in longevity (112) and also enhances the autophagy function in protein degradation by increasing the transcription of several autophagy-related genes, including Atg8/MAPLC3, Atg6, Atg12 and Vps34 (113). The tumor suppressor P53 not only induces autophagy in a damage-mediated autophagy modulator (DRAM)-dependent manner that is essential for P53-mediated programmed cell death-apoptosis (114), but also slows down ageing and reinforces cancer resistance through coordinating with the alternative reading frame protein of Cdkn2a locus (Arf) in p53 and arf increased mice (115). Furthermore, the reactive oxygen species (ROS), which induces oxidative damage of cytosolic materials, regulates both ageing and autophagy (110). Thus, the question to be solved in longevity and autophagy that displays multiple signaling crosstalk concentrates on how to handle unneeded macromolecules and organelles, a determinant of ageing.

\section{Conclusion}

Recent studies have made great progress in uncovering autophagy, which affects both cell survival and cell death. Many proteins that are assembled to form the autophagic machinery have been identified, whereas the complicated regulatory system of autophagy controlled by multiple inhibitory and stimulatory factors involved in many signaling pathway is far from being fully understood. From current knowledge we know that both constructive or basal levels and induced autophagy are important for maintaining cellular metabolism and promoting mammalian health. However, in certain settings, autophagy's protective role may contribute to protecting cells that should die, or its over-activity may result in the death of 
cells that should live. The impairment of autophagy clearance for intracellular proteins and organelles is associated with the pathogenesis of several diseases, such as neurodegeneration, Parkinson's diseases and ageing. Therefore, targeting autophagy may be a potential strategy for the treatment of these diseases. Yet, in the manipulation of autophagy for therapeutic interventions, we should consider the type and progression of the diseases because autophagy may act as a stimulator or a suppressor, such as in cancer. Also, because of the dual functions in cytoprotection and cell death, further research is needed to clarify a more detailed understanding of how autophagy influences different types of human diseases.

\section{References}

1. Levine B and Klionsky DJ: Development by self digestion: molecular mechanisms and biological functions of autophagy. Dev Cell 6: 463-447, 2004.

2. Klionsky DJ: The molecular machinery of autophagy: unanswered questions. J Cell Sci 118: 7-18, 2005.

3. Massey AC, Zhang C and Cuervo AM: Chaperone-mediated autophagy in aging and disease. Curr Top Dev Biol 73: 205-235, 2006

4. Huang WP and Klionsky DJ: Autophagy in yeast: a review of the molecular machinery. Cell Struct Funct 27: 409-420, 2002.

5. Petiot A, Ogier-Denis E, Blommaart EF, Meijer AJ and Codogno P: Distinct classes of phosphatidylinositol 3'-kinases are involved in signaling pathways that control macroautophagy in HT-29 cells. J Biol Chem 275: 992-998, 2000.

6. Pattingre S, Espert L, Biard-Piechaczyk $\mathrm{M}$ and Codogno P: Regulation of macroautophagy by mTOR and Beclin 1 complexes. Biochimie 90: 313-323, 2008 .

7. Nicklin P, Bergman P, Zhang B, Triantafellow E, Wang H, Nyfeler B, Yang H, Hild M, Kung C, Wilson C, Myer VE, MacKeigan JP, Porter JA, Wang YK, Cantley LC, Finan PM and Murphy LO: Bidirectional transport of amino acids regulates mTOR and autophagy. Cell 136: 521-534, 2009.

8. Sancak Y, Peterson TR, Shaul YD, Lindquist RA, Thoreen CC, Bar-Peled L and Sabatini DM: The Rag GTPases bind raptor and mediate amino acid signaling to mTORC1. Science 320: 1496-1501, 2008.

9. Wu YT, Tan HL, Huang Q, Ong CN and Shen HM: Activation of the PI3K-Akt-mTOR signaling pathway promotes necrotic cell death via suppression of autophagy. Autophagy 5: 824-834, 2009.

10. Ganley IG, Lam du H, Wang J, Ding X, Chen S and Jiang X: ULK1.ATG13.FIP200 complex mediates mTOR signaling and is essential for autophagy. J Biol Chem 284: 12297-12305, 2009.

11. Jung CH, Jun CB, Ro SH, Kim YM, Otto NM, Cao J, Kundu M and Kim DH: ULK-Atg13-FIP200 complexes mediate mTOR signaling to the autophagy machinery. Mol Biol Cell 20: 1992-2003, 2009.

12. Hosokawa N, Hara T, Kaizuka T, Kishi C, Takamura A, Miura Y, Iemura S, Natsume T, Takehana K, Yamada N, Guan JL, Oshiro N and Mizushima N: Nutrient-dependent mTORC1 association with the ULK1-Atg13-FIP200 complex required for autophagy. Mol Biol Cell 20: 1981-1991, 2009.

13. Hosokawa N, Sasaki T, Iemura SI, Natsume T, Hara T and Mizushima N: Atg101, a novel mammalian autophagy protein interacting with Atg13. Autophagy 5: 973-979, 2009.

14. Levine B and Kroemer G: Autophagy in the pathogenesis of disease. Cell 132: 27-42, 2008

15. Kawamata T, Kamada Y, Kabeya Y, Sekito T and Ohsumi Y: Organization of the pre-autophagosomal structure responsible for autophagosome formation. Mol Biol Cell 19: 2039-2050, 2008.

16. Suzuki K, Kubota Y, Sekito T and Ohsumi Y: Hierarchy of Atg proteins in pre-autophagosomal structure organization. Genes Cells 12: 209-218, 2007

17. He C, Baba M and Klionsky DJ: Double duty of Atg9 selfassociation in autophagosome biogenesis. Autophagy 5: 385-387, 2009.

18. He C, Baba M, Cao Y and Klionsky DJ: Self-interaction is critical for Atg9 transport and function at the phagophore assembly site during autophagy. Mol Biol Cell 19: 5506-5516, 2008.

19. Fass E, Amar N and Elazar Z: Identification of essential residues for the C-terminal cleavage of the mammalian LC3: a lesson from yeast Atg8. Autophagy 3: 48-50, 2007.
20. Komatsu M, Tanida I, Ueno T, Ohsumi M, Ohsumi Y and Kominami E: The C-terminal region of an Apg $7 \mathrm{p} / \mathrm{Cvt} 2 \mathrm{p}$ is required for homodimerization and is essential for its E1 activity and E1E2 complex formation. J Biol Chem 276: 9846-9854, 2001

21. Fujita N, Itoh T, Omori H, Fukuda M, Noda T and Yoshimori T: The Atg16L complex specifies the site of LC3 lipidation for membrane biogenesis in autophagy. Mol Biol Cell 19: 2092-2100, 2008.

22. Noda T, Fujita N and Yoshimori T: The late stages of autophagy: how does the end begin? Cell Death Differ 16: 984-990, 2009.

23. Kuma A, Matsui M and Mizushima N: LC3, an autophagosome marker, can be incorporated into protein aggregates independent of autophagy: caution in the interpretation of LC3 localization. Autophagy 3: 323-328, 2007.

24. Saftig P, Beertsen W and Eskelinen EL: LAMP-2: a control step for phagosome and autophagosome maturation. Autophagy 4: 510-512, 2008.

25. Cao Y, Espinola JA, Fossale E, Massey AC, Cuervo AM, MacDonald ME and Cotman SL: Autophagy is disrupted in a knock-in mouse model of juvenile neuronal ceroid lipofuscinosis. J Biol Chem 281: 20483-20493, 2006.

26. Jäger S, Bucci C, Tanida I, Ueno T, Kominami E, Saftig P and Eskelinen EL: Role for Rab7 in maturation of late autophagic vacuoles. Cell Sci 117: 4837-4848, 2004

27. Hara T, Nakamura K, Matsui M, Yamamoto A, Nakahara Y, Suzuki-Migishima R, Yokoyama M, Mishima K, Saito I, Okano H and Mizushima N: Suppression of basal autophagy in neural cells causes neurodegenerative disease in mice. Nature 441: 885-889, 2006.

28. Bjørkøy G, Lamark T, Brech A, Outzen H, Perander M, Overvatn A, Stenmark H and Johansen T: p62/SQSTM1 forms protein aggregates degraded by autophagy and has a protective effect on huntingtin-induced cell death. J Cell Biol 171: 603-614, 2005.

29. Bence NF, Sampat RM and Kopito RR: Impairment of the ubiquitin-proteasome system by protein aggregation. Science 292: 1552-1555, 2001

30. Ichimura Y, Kominami E, Tanaka K and Komatsu M: Selective turnover of p62/A170/SQSTM1 by autophagy. Autophagy 4: 1063-1066, 2008.

31. Kim I, Rodriguez-Enriquez S and Lemasters JJ: Selective degradation of mitochondria by mitophagy. Arch Biochem Biophys 462: 245-253, 2007.

32. Sandoval H, Thiagarajan P, Dasgupta SK, Schumacher A, Prchal JT, Chen M and Wang J: Essential role for Nix in autophagic maturation of erythroid cells. Nature 454: 232-235, 2008.

33. Kim PK, Hailey DW, Mullen RT and Lippincott-Schwartz J: Ubiquitin signals autophagic degradation of cytosolic proteins and peroxisomes. Proc Natl Acad Sci USA 105: 20567-20574, 2008.

34. Kraft C, Deplazes A, Sohrmann M and Peter M: Mature ribosomes are selectively degraded upon starvation by an autophagy pathway requiring the Ubp3p/Bre5p ubiquitin protease. Nat Cell Biol 10: 602-610, 2008.

35. Singh R, Kaushik S, Wang Y, Xiang Y, Novak I, Komatsu M, Tanaka K, Cuervo AM and Czaja MJ: Autophagy regulates lipid metabolism. Nature 458: 1131-1135, 2009.

36. Levine B and Yuan J: Autophagy in cell death: an innocent convict? J Clin Invest 115: 2679-2688, 2005.

37. Yorimitsu T, Nair U, Yang Z and Klionsky DJ: Endoplasmic reticulum stress triggers autophagy. J Biol Chem 281: 30299-30304, 2006.

38. Lum JJ, DeBerardinis RJ and Thompson CB: Autophagy in metazoans: cell survival in the land of plenty. Nat Rev Mol Cell Biol 6: 439-448, 2005

39. Maiuri MC, Zalckvar E, Kimchi A and Kroemer G: Self-eating and self-killing: crosstalk between autophagy and apoptosis. Nat Rev Mol Cell Biol 8: 741-752, 2007.

40. Lum JJ, Bauer DE, Kong M, Harris MH, Li C, Lindsten T and Thompson CB: Growth factor regulation of autophagy and cell survival in the absence of apoptosis. Cell 120: 237-248, 2005

41. Qu X, Zou Z, Sun Q, Luby-Phelps K, Cheng P, Hogan RN, Gilpin C and Levine B: Autophagy gene-dependent clearance of apoptotic cells during embryonic development. Cell 128: 931-946, 2007.

42. Kuma A, Hatano M, Matsui M, Yamamoto A, Nakaya $H$ Yoshimori T, Ohsumi Y, Tokuhisa T and Mizushima N: The role of autophagy during the early neonatal starvation period. Nature 432: 1032-1036, 2004.

43. Komatsu M, Waguri S, Ueno T, Iwata J, Murata S, Tanida I, Ezaki J, Mizushima N, Ohsumi Y, Uchiyama Y, Kominami E, Tanaka $\mathrm{K}$ and Chiba T: Impairment of starvation-induced and constitutive autophagy in Atg7-deficient mice. J Cell Biol 169: $425-434,2005$ 
44. Tsukamoto S, Kuma A, Murakami M, Kishi C, Yamamoto A and Mizushima N: Autophagy is essential for preimplantation development of mouse embryos. Science 321: 117-120, 2008.

45. Münz C: Enhancing immunity through autophagy. Annu Rev Immunol 27: 423-449, 2009.

46. Nakagawa I, Amano A, Mizushima N, Yamamoto A, Yamaguchi H, Kamimoto T, Nara A, Funao J, Nakata M, Tsuda K, Hamada S and Yoshimori T: Autophagy defends cells against invading group A Streptococcus. Science 306: 1037-1040, 2004.

47. Paludan C, Schmid D, Landthaler M, Vockerodt M, Kube D, Tuschl T and Münz C: Endogenous MHC class II processing of a viral nuclear antigen after autophagy. Science 307: 593-596, 2005.

48. Gutierrez MG, Master SS, Singh SB, Taylor GA, Colombo MI and Deretic V: Autophagy is a defense mechanism inhibiting BCG and Mycobacterium tuberculosis survival in infected macrophages. Cell 119: 753-766, 2004.

49. Jagannath C, Lindsey DR, Dhandayuthapani S, Xu Y, Hunter RL Jr and Eissa NT: Autophagy enhances the efficacy of BCG vaccine by increasing peptide presentation in mouse dendritic cells. Nat Med 15: 267-276, 2009.

50. Scarlatti F, Granata R, Meijer AJ and Codogno P: Does autophagy have a license to kill mammalian cells? Cell Death Differ 16: 12-20, 2009.

51. Shimizu S, Kanaseki T, Mizushima N, Mizuta T, Arakawa-Kobayashi S, Thompson CB and Tsujimoto Y: Role of Bcl-2 family proteins in a non-apoptotic programmed cell death dependent on autophagy genes. Nat Cell Biol 6: 1221-1228, 2004.

52. Pattingre S, Tassa A, Qu X, Garuti R, Liang XH, Mizushima N, Packer M, Schneider MD and Levine B: Bcl-2 antiapoptotic proteins inhibit Beclin 1-dependent autophagy. Cell 122: 927-939, 2005.

53. Gude DR, Alvarez SE, Paugh SW, Mitra P, Yu J, Griffiths R, Barbour SE, Milstien S and Spiegel S: Apoptosis induces expression of sphingosine kinase 1 to release sphingosine-1-phosphate as a 'come-and-get-me' signal. FASEB J 22: 2629-2638, 2008.

54. Lavieu G, Scarlatti F, Sala G, Carpentier S, Levade T, Ghidoni R, Botti J and Codogno P: Regulation of autophagy by sphingosine kinase 1 and its role in cell survival during nutrient starvation. J Biol Chem 281: 8518-8527, 2006.

55. Maiuri MC, Malik SA, Morselli E, Kepp O, Criollo A, Mouchel PL, Carnuccio R and Kroemer G: Stimulation of autophagy by the p53 target gene Sestrin2. Cell Cycle 8: 1571-1576, 2009.

56. Vicencio JM, Ortiz C, Criollo A, et al: The inositol 1,4,5trisphosphate receptor regulates autophagy through its interaction with Beclin 1. Cell Death Differ 16: 1006-1017, 2009.

57. Maiuri MC, Le Toumelin G, Criollo A, Rain JC, Gautier F, Juin P, Tasdemir E, Pierron G, Troulinaki K, Tavernarakis N, Hickman JA, Geneste $\mathrm{O}$ and Kroemer G: Functional and physical interaction between Bcl-XL and a BH3-like domain in Beclin-1. EMBO J 26: 2527-2539, 2007.

58. Mizushima N, Levine B, Cuervo AM and Klionsky DJ: Autophagy fights disease through cellular self-digestion. Nature 451: 1069-1075, 2008.

59. Sarkar S and Rubinsztein DC: Small molecule enhancers of autophagy for neurodegenerative diseases. Mol Biosyst 4: 895-901, 2008

60. Honson NS, Johnson RL, Huang W, Inglese J, Austin CP and Kuret J: Differentiating Alzheimer disease-associated aggregates with small molecules. Neurobiol Dis 28: 251-260, 2007.

61. Williams A, Jahreiss L, Sarkar S, Saiki S, Menzies FM, Ravikumar B and Rubinsztein DC: Aggregate-prone proteins are cleared from the cytosol by autophagy: therapeutic implications. Curr Top Dev Biol 76: 89-101, 2006.

62. Boland B, Kumar A, Lee S, Platt FM, Wegiel J, Yu WH and Nixon RA: Autophagy induction and autophagosome clearance in neurons: relationship to autophagic pathology in Alzheimer's disease. J Neurosci 28: 6926-6937, 2008.

63. Lee JA and Gao FB: Inhibition of autophagy induction delays neuronal cell loss caused by dysfunctional ESCRT-III in frontotemporal dementia. J Neurosci 29: 8506-8511, 2009.

64. Ravikumar B, Vacher C, Berger Z, Davies JE, Luo S, Oroz LG, Scaravilli F, Easton DF, Duden R, O'Kane CJ and Rubinsztein DC: Inhibition of mTOR induces autophagy and reduces toxicity of polyglutamine expansions in fly and mouse models of Huntington disease. Nature Genet 36: 585-595, 2004.

65. Sarbassov DD, Ali SM and Sabatini DM: Growing roles for the mTOR pathway. Curr Opin Cell Biol 17: 596-603, 2005.

66. Bissler JJ, McCormack FX, Young LR, Elwing JM, Chuck G, Leonard JM, Schmithorst VJ, Laor T, Brody AS, Bean J, Salisbury S and Franz DN: Sirolimus for angiomyolipoma in tuberous sclerosis complex or lymphangioleiomyomatosis. N Engl J Med 358: 140-151, 2008.
67. Sarkar S, Perlstein EO, Imarisio S, Pineau S, Cordenier A, Maglathlin RL, Webster JA, Lewis TA, O'Kane CJ, Schreiber SL and Rubinsztein DC: Small molecules enhance autophagy and reduce toxicity in Huntington's disease models. Nature Chem Biol 3: 331-338, 2007.

68. Williams A, Sarkar S, Cuddon P, Ttofi EK, Saiki S, Siddiqi FH, Jahreiss L, Fleming A, Pask D, Goldsmith P, O'Kane CJ, Floto RA and Rubinsztein DC: Novel targets for Huntington's disease in an mTOR-independent autophagy pathway. Nat Chem Biol 4: 295-305, 2008

69. Yu WH, Cuervo AM, Kumar A, et al: Macroautophagy - a novel Beta-amyloid peptide-generating pathway activated in Alzheimer's disease. J Cell Biol 171: 87-98, 2005.

70. Kamimoto T, Shoji S, Hidvegi T, Mizushima N, Umebayashi K, Perlmutter DH and Yoshimori T: Intracellular inclusions containing mutant $\alpha-1$ antitrypsin $Z$ are propagated in the absence of autophagic activity. J Biol Chem 281: 4467-4476, 2006.

71. Kostin S, Pool L, Elsasser A, Hein S, Drexler HC, Arnon E, Hayakawa Y, Zimmermann R, Bauer E, Klovekorn WP and Schaper J: Myocytes die by multiple mechanisms in failing human hearts. Circ Res 92: 715-724, 2003.

72. Weekes J, Morrison K, Mullen A, Wait R, Barton P and Dunn MJ: Hyperubiquitination of proteins in dilated cardiomyopathy. Proteomics 3: 208-216, 2003.

73. Heling A, Zimmermann R, Kostin S, Maeno Y, Hein S, Devaux B, Bauer E, Klovekorn WP, Schlepper M, Schaper W and Schaper J: Increased expression of cytoskeletal, linkage, and extracellular proteins in failing human myocardium. Circ Res 86: 846-853, 2000.

74. Tannous P, Zhu H, Nemchenko A, Berry JM, Johnstone JL, Shelton JM, Miller FJ Jr, Rothermel BA and Hill JA: Intracellular protein aggregation is a proximal trigger of cardiomyocyte autophagy. Circulation 117: 3070-3078, 2008.

75. Nakai A, Yamaguchi O, Takeda T, Higuchi Y, Hikoso S, Taniike M, Omiya S, Mizote I, Matsumura Y, Asahi M, Nishida K, Hori M, Mizushima $\mathrm{N}$ and Otsu K: The role of autophagy in cardiomyocytes in the basal state and in response to hemodynamic stress. Nat Med 13: 619-624, 2007.

76. Zhu H, Tannous P, Johnstone JL, Kong Y, Shelton JM, Richardson JA, Le V, Levine B, Rothermel BA and Hill JA: Cardiac autophagy is a maladaptive response to hemodynamic stress. J Clin Invest 117: 1782-1793, 2007.

77. Rothermel BA and Hill JA: Autophagy in load-induced heart disease. Circ Res 103: 1363-1369, 2008.

78. Nishino I, Fu J, Tanji K, et al: Primary LAMP-2 deficiency causes X-linked vacuolar cardiomyopathy and myopathy (Danon disease). Nature 406: 906-910, 2000.

79. Raben N, Hill V, Shea L, Takikita S, Baum R, Mizushima N, Ralston E and Plotz P: Suppression of autophagy in skeletal muscle uncovers the accumulation of ubiquitinated proteins and their potential role in muscle damage in Pompe disease. Hum Mol Genet 17: 3897-3908, 2008

80. Ramachandran N, Munteanu I, Wang P, et al: VMA21 deficiency causes an autophagic myopathy by compromising V-ATPase activity and lysosomal acidification. Cell 137: 235-246, 2009.

81. Eisenberg-Lerner A and Kimchi A: The paradox of autophagy and its implication in cancer etiology and therapy. Apoptosis 14: 376-391, 2009

82. Liang XH, Jackson S, Seaman M, Brown K, Kempkes B, Hibshoosh $\mathrm{H}$ and Levine B: Induction of autophagy and inhibition of tumorigenesis by Beclin 1. Nature 402: 672-676, 1999.

83. Miracco C, Cosci E, Oliveri G, Luzi P, Pacenti L, Monciatti I, Mannucci S, De Nisi MC, Toscano M, Malagnino V, Falzarano SM, Pirtoli L and Tosi P: Protein and mRNA expression of autophagy gene Beclin 1 in human brain tumours. Int J Oncol 30: 429-436, 2007.

84. Qu X, Yu J, Bhagat G, Furuya N, Hibshoosh H, Troxel A, Rosen J, Eskelinen EL, Mizushima N, Ohsumi Y, Cattoretti G and Levine B: Promotion of tumorigenesis by heterozygous disruption of the beclin 1 autophagy gene. J Clin Invest 112: 1809-1820, 2003.

85. Yue Z, Jin S, Yang C, Levine AJ and Heintz N: Beclin 1, an autophagy gene essential for early embryonic development, is a haploin sufficient tumor suppressor. Proc Natl Acad Sci USA 100: 15077-15082, 2003

86. Kang MR, Kim MS, Oh JE, Kim YR, Song SY, Kim SS, Ahn CH, Yoo NJ and Lee SH: Frameshift mutations of autophagy-related genes ATG2B, ATG5, ATG9B and ATG12 in gastric and colorectal cancers with microsatellite instability. J Pathol 217: 702-706, 2009 
87. Mariño G, Salvador-Montoliu N, Fueyo A, Knecht E, Mizushima N and López-Otín C: Tissue-specific autophagy alterations and increased tumorigenesis in mice deficient in Atg4C/autophagin-3. J Biol Chem 282: 18573-18583, 2007.

88. Maiuri MC, Tasdemir E, Criollo A, Morselli E, Vicencio JM, Carnuccio R and Kroemer G: Control of autophagy by oncogenes and tumor suppressor genes. Cell Death Differ 16: 87-93, 2009.

89. Tambe Y, Yamamoto A, Isono T, Chano T, Fukuda M and Inoue $\mathrm{H}$ : The drs tumor suppressor is involved in the maturation process of autophagy induced by low serum. Cancer Lett 283: 74-83, 2009.

90. Mathew R, Karp CM, Beaudoin B, Vuong N, Chen G, Chen HY, Bray K, Reddy A, Bhanot G, Gelinas C, Dipaola RS, Karantza-Wadsworth V and White E: Autophagy suppresses tumorigenesis through elimination of p62. Cell 137: 1062$1075,2009$.

91. Mathew R, Karantza-Wadsworth V and White E: Role of autophagy in cancer. Nat Rev Cancer 7: 961-967, 2007.

92. Milani M, Rzymski T, Mellor HR, Pike L, Bottini A, Generali D and Harris AL: The role of ATF4 stabilization and autophagy in resistance of breast cancer cells treated with Bortezomib. Cancer Res 69: 4415-4423, 2009.

93. Vazquez-Martin A, Oliveras-Ferraros C and Menendez JA: Autophagy facilitates the development of breast cancer resistance to the anti-HER2 monoclonal antibody trastuzumab. PLoS One 4: E6251, 2009.

94. Carew JS, Medina EC, Esquivel JA 2nd, Mahalingam D, Swords R, Kelly K, Zhang H, Huang P, Mita AC, Mita MM, Giles FJ and Nawrocki ST: Autophagy inhibition enhances vorinostat-induced apoptosis via ubiquitinated protein accumulation. J Cell Mol Med (In press).

95. Qadir MA, Kwok B, Dragowska WH, To KH, Le D, Bally MB and Gorski SM: Macroautophagy inhibition sensitizes tamoxifen-resistant breast cancer cells and enhances mitochondrial depolarization. Breast Cancer Res Treat Dec 112: 389-403, 2008.

96. Apel A, Herr I, Schwarz H, Rodemann HP and Mayer A: Blocked autophagy sensitizes resistant carcinoma cells to radiation therapy. Cancer Res 68: 1485-1494, 2008.

97. Ogawa M, Yoshimori T, Suzuki T, Sagara H, Mizushima N and Sasakawa C: Escape of intracellular Shigella from autophagy. Science 307: 727-731, 2005

98. Birmingham CL, Canadien V, Kaniuk NA, Steinberg BE, Higgins DE and Brumell JH: Listeriolysin O allows Listeria monocytogenes replication in macrophage vacuoles. Nature 451: 350-354, 2008 .

99. Orvedahl A, Alexander D, Tallóczy Z, Sun Q, Wei Y, Zhang W, Burns D, Leib DA and Levine B: HSV-1 ICP34.5 confers neurovirulence by targeting the Beclin 1 autophagy protein. Cell Host Microbe 1: 23-35, 2007

100. Ku B, Woo JS, Liang C, Lee KH, Hong HS, E X, Kim KS, Jung JU and Oh BH: Structural and biochemical bases for the inhibition of autophagy and apoptosis by viral BCL-2 of murine $\gamma$-herpesvirus 68. PLoS Pathog 4: E25, 2008.
101. Rioux JD, Xavier RJ, Taylor KD, et al: Genome-wide association study identifies new susceptibility loci for Crohn disease and implicates autophagy in disease pathogenesis. Nat Genet 39: 596-604, 2007.

102. McCarroll SA, Huett A, Kuballa P, et al: Deletion polymorphism upstream of IRGM associated with altered IRGM expression and Crohn's disease. Nat Genet 40: 1107-1112, 2008.

103. Hampe J, Franke A, Rosenstiel P, et al: A genome-wide association scan of nonsynonymous SNPs identifies a susceptibility variant for Crohn disease in ATG16L1. Nat Genet 39: 207-211, 2007

104. Parkes M, Barrett JC, Prescott NJ, et al: Sequence variants in the autophagy gene IRGM and multiple other replicating loci contribute to Crohn's disease susceptibility. Nat Genet 39: 830-832, 2007

105. Cuervo AM, Bergamini E, Brunk UT, Dröge W, Ffrench M and Terman A: Autophagy and aging: the importance of maintaining 'clean' cells. Autophagy 1: 131-140, 2005

106. Del Roso A, Vittorini S, Cavallini G, Donati A, Gori Z, Masini M, Pollera $\mathrm{M}$ and Bergamini E: Ageing-related changes in the in vivo function of rat liver macroautophagy and proteolysis. Exp Gerontol 38: 519-527, 2003

107. Kenyon C: The plasticity of aging: insights from long-lived mutants. Cell 120: 449-460, 2005.

108. Bergamini E: Targets for antiageing drugs. Expert Opin Ther Targets 9: 77-82, 2005.

109. Donati A, Ventruti A, Cavallini G, Masini M, Vittorini S, Chantret I, Codogno P and Bergamini E: In vivo effect of an antilipolytic drug (3,5'-dimethylpyrazole) on autophagic proteolysis and autophagy-related gene expression in rat liver. Biochem Biophys Res Commun 366: 786-792, 2008.

110. Vellai T: Autophagy genes and ageing. Cell Death Differ 16: 94-102, 2009.

111. Holzenberger M, Dupont J, Ducos B, Leneuve P, Geloen A, Even PC, Cervera P and Le Bouc Y: IGF-1 receptor regulates lifespan and resistance to oxidative stress in mice. Nature 421: 125-126, 2003.

112. Salih DA and Brunet A: FoxO transcription factors in the maintenance of cellular homeostasis during aging. Curr Opin Cell Biol 20: 126-136, 2008.

113.Zhao J, Brault JJ, Schild A, Cao P, Sandri M, Schiaffino S, Lecker SH and Goldberg AL: FoxO3 co-ordinately activates protein degradation by the autophagic/lysosomal and proteasomal pathways in atrophying muscle cells. Cell Metab 6: 472-483, 2007.

114. Crighton D, Wilkinson S, O'Prey J, Syed N, Smith P, Harrison PR, Gasco M, Garrone O, Crook T and Ryan KM: DRAM, a p53induced modulator of autophagy, is critical for apoptosis. Cell 126: 121-134, 2006.

115. Matheu A, Maraver A, Klatt P, Flores I, Garcia-Cao I, Borras C, Flores JM, Viña J, Blasco MA and Serrano M: Delayed ageing through damage protection by the Arf/p53 pathway. Nature 448: 375-380, 2007. 